
and Biotechnology.

Author(s):

P. Chris Hammel, MST-10

Griselda Hernández, Jill Trewhella,

Clifford J. Unkefer, CST-4

Donald K. Blumenthal, U. of Utah Michael A. Kennedy, PNNL

Gregory J. Moore, Wayne State U.

Los Alamos National Laboratory, an affirmative action/equal opportunity employer, is operated by the University of California for the U.S. Department of Energy under contract W-7405-ENG-36. By acceptance of this articie, the publisher recognizes that the U.S.

Government retains a nonexclusive, royalty-free license to publish or reproduce the published form of this contribution, or to allow others to do so, for U.S. Government purposes. Los Alamos National Laboratory requests that the publisher identify this article as work performed under the auspices of the U.S. Department of Energy. The Los Alamos National Laboratory strongly supports academic freedom and a researcher's right to publish; as an institution, however, the Laboratory does not endorse the viewpoint of a publication or guarantee its technical correctness. 


\section{DISCLAIMER}

This report was prepared as an account of work sponsored by an agency of the United States Government. Neither the United States Government nor any agency thereof, nor any of their employees, makes any wartanty, express or implied, or assumes any legal liability or responsibility for the accuracy, completeness, or usefulness of any information, apparatus, product, or process disclosed, or represents that its use would not infringe privately owned rights. Reference herein to any specific commercial product, process, or service by trade name, trademark, manufacturer, or otherwise does not necessarily constitute or imply its endorsement, recommendation, or favoring by the United States Government or any agency thereof. The views and opinions of authors expressed herein do not necessarily state or reflect those of the United States Government or any agency thereof. 


\section{DISCLAIMER}

Portions of this document may be illegible in electronic image products. Images are produced from the best available original document. 


\title{
Advanced NMR Technology for Bioscience and Biotechnology.
}

\author{
P. Chris Hammel, Griselda Hernández, Jill Trewhella*, and Clifford J. Unkefer \\ Los Alamos National Laboratory \\ Donald K. Blumenthal \\ University of Utah \\ Michael A. Kennedy \\ Pacific Northwest National Laboratory \\ Gregory J. Moore \\ Wayne State University
}

\begin{abstract}
This is the final report of a three-year, Laboratory Directed Research and Development (LDRD) project at the Los Alamos National Laboratory (LANL). NMR plays critical roles in bioscience and biotechnology in both imaging and structure determination. NMR is limited, however, by the inherent low sensitivity of the NMR experiment and the demands for spectral resolution required to study biomolecules. We addressed both of these issues by working on the development of NMR force microscopy for molecular imaging, and high field NMR with isotope labeling to overcome limitations in the size of biomolecules that can be studied using NMR. A novel rf coil design for NMR force microscopy was developed that increases the limits of sensitivity in magnetic resonance detection for imaging, and we demonstrated sub-surface spatial imaging capabilities. We also made advances in the miniaturization of two critical NMR force microscope components. We completed high field NMR and isotope labeling studies of a muscle protein complex which is responsible for regulating muscle contraction and is too large for study using conventional NMR approaches.
\end{abstract}

\section{Background and Research Objectives}

NMR technologies continue to play critical roles in bioscience and biotechnology in both imaging and molecular structure determination. Two issues limit the application of NMR in many important problems in bioscience: 1) the inherent low sensitivity of NMR experiments and 2) the need for greater resolution. This project addresses both of these issues. In the area of structure determination high-resolution high-field NMR, in combination with stable isotope labeling, has seen dramatic changes in the past five years. These changes have resulted in a growing data base of three-dimensional structures of biomolecules in solution that are of the same quality as those obtained from $\mathrm{x}$-ray

*Principal Investigator, e-mail: jtrewhella@lanl.gov 
crystallography. NMR methods also now offer the potential for highly sensitive, chemically specific imaging of biological systems at high resolution using force microscopy detection. The principal focus of this project is on the development of enhanced capabilities for NMR technologies at Los Alamos including very high field NMR applications to molecular structure determination, and NMR force microscopy development.

Magnetic resonance force microscopy (MRFM): MRFM (Publication 2) has the capability to perform direct, three dimensional, chemically specific imaging of biological structures with a noncontacting/nondestructive probe. In addition it has the capability to probe in a well-defined sub-surface volume. By using mechanical detection, it offers greatly enhanced sensitivity over conventional magnetic resonance imaging (MRI). If developed to its theoretical potential MRFM provides the promise of imaging single-copy biomolecules, e.g. on cell surfaces. At this time there is a dearth of structural information on membrane bound proteins including receptors that are key players for drug design and environmental biotechnologies, as well as those that are primary targets for chemical and biological warfare agents. These systems have been resistant to study using conventional structural methodologies for a variety of reasons. MRFM could provide critical breakthroughs for studying these important systems.

Very high field NMR applications to molecular structure determination: Over the past decade NMR spectroscopy has emerged as a major player in high resolution biomolecular structure determination as a result of technological advances in available magnetic field strengths, increases in sensitivity, and the use of isotope labels for increasing spectral resolution. Since the first complete three-dimensional NMR structure of a protein appeared in 1984 (bull seminal protease inhibitor IIA), there have been $\sim 200$ NMR protein structures published (compared with $\sim 600$ x-ray crystal structures since the first crystallography protein structure (myoglobin) in the 1950's). NMR techniques have opened an important doorway for the structural biologist, giving complete threedimensional solution structures for biomolecules. This is particularly important for determining structures of complexes between proteins as well as protein/DNA (or RNA) complexes that have proved difficult to crystallize.

The major limitations to NMR solution structure determination in biomolecules lie in the size of the systems that can be studied. Two factors contribute to this size limitation. First, larger molecules tumble slowly, which effectively increases the spin-spin lattice relaxation rate $\left(T_{2}\right)$ increasing the resonance linewidth. In addition, the increased linewidth coupled with the fact that the absolute number of protons increases in direct proportion to molecular weight results in spectral overlap problems for large bioploymers. Two approaches will be required to overcome the spectral overlap problem at higher molecular 
weights. First NMR spectral resolution is directly related to the applied magnetic field, so increasing the field increases the resolution and allows larger bioploymers to be studied. The arrival of the first $750 \mathrm{MHz}$ spectrometer and the promise of a $\mathrm{GHz}$ class NMR spectrometer at Pacific Northwest National Laboratory (PNNL) represent a significant gain in the available field strengths for structural NMR. The second critical problem with approaching large molecules arises from line broadening due to increased spin-spin $\left(T_{2}\right)$ relaxation rates that result from the slower tumbling of larger molecules. Because of the low gyromagnetic ratio of deuterium, deuterium substitution for hydrogen can alleviate the effects of line broadening by eliminating dipolar interactions with, as well as between, hydrogen nuclei. Deuteration can also be used to eliminate effects of spin diffusion between hydrogen nuclei.

\section{Importance to LANL's Science and Technology Base and National R\&D Needs}

Biomolecular structure determination, both in solution and on surfaces, is a key capability for developments in molecular medicine including rational drug design and biotechnologies that utilize biological structures for technological applications.

Applications in the development of novel self-assembling biomolecular materials for use as sensors or in energy storage and transfer, and in the development of biosystems for remediation and waste treatment, depend upon detailed three-dimensional structural information at the highest resolution attainable so that the chemistry and physics of these systems can be understood and used. Pushing solution structure methods to larger and more complex systems, and developing the capability for structure determination of singlecopy molecules on surfaces (for example) are critical technologies for the future. High resolution NMR with stable isotopes and MRFM are pushing at the forefront in these areas. This project builds upon Los Alamos capabilities in structural biology, stable isotope chemistry, high field NMR, fiber optics, and nanotechnology; it directly supports our Bioscience and Biotechnology core competency as well as the Laboratory's Genome and Beyond tactical goal.

\section{Scientific Approach and Accomplishments}

Magnetic resonance force microscopy: We have made significant technical progress on the magnetic resonance force microscope technology development (Publications 2 and 5). Specifically, we designed and completed construction of a magnetic resonance force microscope for detecting electron spin resonance signals in vacuum. We have thus far achieved detection sensitivities of better than $10^{-10}$ electron spins, a full two orders of magnitude better than that obtainable with conventional inductive detection technologies. 
We have also designed an NMR force microscope device. We designed and built a fiber optic interferometry sensor for the force microscope instruments. This sensor is compatible for use with both vacuum and low temperature measurements. We have demonstrated the sensitivity of this system to be $0.002 \AA$ per root $\mathrm{Hz}$. With this sensor we successfully measured the mechanical resonance in cantilevers driven by thermal noise at an amplitude of $5 \AA$ with a signal to noise of approximately 20 .

Verv high field NMR applications to molecular structure determination: We have established a critical collaboration for this part of the project with Paul Ellis and Michael Kennedy at the Environmental Molecular Sciences Laboratory at PNNL. The 750-MHz NMR spectrometer at PNNL was the first operational in the US, and they are now awaiting delivery (Spring, 1998) from Oxford Instruments the first $>900-\mathrm{MHz}$ high-field magnet for NMR spectroscopy (Publications 1 and 7). With our PNNL collaborators we have demonstrated the applicability of new stable isotope labeling strategies for studying the structures of biomolecular complexes. Specifically, we have utilized NMR spectroscopy and deuterium labeling to study the conformation of the troponin I inhibitory peptide ( $\operatorname{TnI}(96-115)$ ) when bound to troponin C (Publications 3,4, and 6). The isotope labeling strategy we used enabled us to measure NMR data from the peptide bound to TnC with a stoichiometric (1:1) ratio of the components. We thus were able to demonstrate that one can effectively use deuteration to facilitate NMR studies of a peptide in a relatively large complex with stoichiometric ratios of the components, thus eliminating the ambiguities that can be associated with the currently popular transferred Nuclear Overhauser Effect (NOE) methods arising from the effects of slow chemical exchange and/or spin diffusion $[1,2,3]$.

The troponin complex functions as a calcium-dependent switch in muscle contraction, regulating the interactions between the interdigitated thick and thin filaments that are proposed to slide past each other during the contraction/relaxation cycle [4]. Troponin has three components: troponin $\mathrm{C}(\mathrm{TnC})$ which binds $\mathrm{Ca}^{2+}$, troponin I (TnI) which inhibits the interactions between the thick and thin filaments, and troponin $\mathrm{T}$ which anchors troponin to the thin filament and is believed to be involved in the transmission of regulatory signals along the thin filament. The crystal structure of $\mathrm{TnC}$ is unusual in that it shows the protein to consist of two globular domains separated by an extended $\alpha$-helix of 7-8 turns [5]. Using neutron and x-ray solution scattering [6] we had previously shown that when complexed with $\mathrm{TnI}$ and $4 \mathrm{Ca}^{2+}, \mathrm{TnC}$ has an extended structure that is very similar to its crystal form, and further that $\mathrm{TnI}$ is even more extended, forming a long, apparently helical structure that encompasses $\mathrm{TnC}$ and passes through the hydrophobic clefts on each globular domain [7]. Our structural model for the complex suggests a mechanism for the "switch" function that involves the binding and release of the helical TnI 
by $\mathrm{TnC}$ via an opening and closing of the hydrophobic cleft in the $\mathrm{N}$-terminal domain of TnC. Validation of this model requires higher resolution structural data on the complex using other approaches. The relatively low solubility and the high molecular weight ( $\sim 38$ $\mathrm{kDa}$ ) of the binary complex of skeletal muscle $\mathrm{TnC} / \mathrm{TnI}$ is such that it is not particularly amenable to high-resolution structural analysis using current NMR technologies, and so this system is ideal for evaluating novel high-field NMR and stable-isotope labeling strategies designed to push the limits of the size and complexity of the systems that can be studied.

Our NMR experiments on deuterated TnC complexed with non-deuterated TnI(96115) revealed that the TnI(96-115) peptide has novel structural characteristics. It gives characteristic NMR signatures of a nascent helix including the $\mathrm{N}$-terminal half of the peptide with an extended conformation for the $\mathrm{C}$-terminal half. The nascent helix has previously been observed and characterized as a transition state in protein folding [8]. In the context of protein folding, the nascent helix is stabilized by helix-stabilizing solvents or by interactions with (or within) proteins. While our studies of $\operatorname{TnI}(96-115)$ indicate it has some nascent helix character, a stable helix is not observed either when the peptide is dissolved by helix-stabilizing solvents or when it is complexed with $\mathrm{TnC}$. Thus it appears that $\mathrm{TnC}$ does not constrain the bound peptide to a single well defined conformation, but rather holds it weakly allowing it to have a set of folded conformations, and the NMR data reflect the population weighted average. This type of binding may be critical to the inhibitory function of this sequence in TnI, which must have sufficient flexibility to bind alternately to $\mathrm{TnC}$ and actin during the contractile cycle.

In addition to providing insights into the mechanism of action of the $\mathrm{TnC} / \mathrm{TnI}$ complex, the results of our studies on $\mathrm{TnI}(96-115)$ bound to $\mathrm{TnC}$ also clearly demonstrate the perils of using transferred NOE (TRNOE) measurements to study the structures of small peptides bound to large proteins. Campbell and Sykes (1991) used TRNOEs to study the C-terminal half of our peptide ( $\operatorname{TnI}(104-115)$ ) bound to TnC. Their experiments were designed to measure NOE data from samples in which the peptide of interest is present in large excess of the protein to which it binds. In the presence of chemical exchange of the ligand between its free and bound states, negative NOEs conveying conformational information of the bound ligand are "transferred" to the free ligand resonances where they are more easily measured because these resonances are much narrower. In this process it is possible, however, that spin diffusion effects arising from the protons in the protein can compromise the interpretation of the transferred NOE in terms of distances within the peptide and hence in terms of the bound peptide structure. Sykes and Campbell interpreted their TRNOE data in terms of the TnI(104-115) sequence 
adopting a helical conformation when bound to $\mathrm{TnC}$. Our experiments with 1:1 TnC:TnI(96-115) show no evidence for such helical turns. These results further demonstrate the importance of isotope labeling strategies for studying biomolecular complexes in solution using high field NMR.

\section{Publications}

1. Cross, T. A., Drobny, G., Trewhella, J. "GHz Nuclear Magnetic Resonance," Stable Isotope Applications in Biomolecular Structure and Mechanisms, Proceedings of the Conference in Santa Fe, New Mexico, pp 315-323, (1994).

2. Hammel, P. C., Zhang, Z., Moore, G. J., Roukes, M. L. "Sub-Surface Imaging with the Magnetic Resonance Force Microscope," Journal of Low Temperature Physics 101, 59 (1995).

3. Hernández, G., Rokop, S. E., Springer, P. A., Unkefer, C. J., Trewhella, J. NMR Studies of Troponin I(96-115): Free in Solution and Bound to Troponin C," Biophysical Journal 70, 384 (1996).

4. Trewhella, J., Hernández, G., Rokop, S. E., Springer, P. A., Unkefer, C. J. "High Field NMR and Stable Isotopes for Studying $\mathrm{Ca}^{2+}$-Binding Proteins and their Complexes," 27th Annual South Easter Magnetic Resonance Conference, Tallahasse FL (1995).

5. Zhang, Z., Hammel, P. C., Moore, G. J. "Application of a Novel rf Coild Design to the Magnetic Resonance Force Microscope," Reviews of Scientific Instruments 67, 3307 (1996).

6. Hernández, G., Kennedy, M. A., Rokop, S. E., Unkefer, C. J., Trewhella, J. "NMR Studies of the Troponin I Inhibitory Peptide (96-115) Bound to Skeletal Muscle Troponin C," in review J. Mol. Biol. (1997).

7. Hoyt, D. W., Zhao, J., Trewhella, J., Kennedy, M. A. "Comparison of Small-Angle $\mathrm{x}$-ray Scattering and Grandient Encoded NMR Diffusion Experiments for Assaying the Aggregation State of Proteins in Solution," to be submitted J. Mag. Res. (1997). 


\section{References}

[1] London, R. E., Perlman, M. E., Davis, D. G. "Relaxation-matrix analysis of the transferred nuclear Overhauser effect for finite exchange rates," J. Magn. Reson. 97, 79-98 (1992).

[2] Zheng, J., Post, C.B. "Protein indirect relaxation effects in exchange-transferred NOESY by a rate-matrix analysis," J. Magn. Reson. B. 101, 262-270 (1993).

[3] Arepalli, S. R., Glaudemans, C. P. J., Daves, G.D., Kovac, P., Bax, A. "Identification of protein-mediated indirect NOE effects in a disaccharide-Fab" complex by transferred ROESY," J. Magn. Reson. B. 106, 195-198 (1995).

[4] Zot, A. S., Potter, J. D. Structural aspects of troponin-tropomyosin regulation of skeletal-muscle contraction. Annu. Rev. Biophys. Biophys. Chem. 16, 535-559 (1987).

[5] Herzberg, O., James, M. N. G. "Structure of the calcium regulatory muscle protein troponin-C at 2.8Ä resolution," Nature 313, 653-659, (1985).

[6] Olah, G. A., Rokop. S. E., Wang, A. C.L., Blechner, S. L., Trewhella, J. "Troponin-I encompasses an extended troponin- $\mathrm{C}$ in the $\mathrm{Ca}^{2+}$ bound complex: $\mathrm{A}$ small-angle $\mathrm{x}$-ray and neutron scattering study," Biochemistry 33, 8233-8239 (1994).

[7] Olah, G. A., Trewhella, J. "A model structure of the muscle protein complex $4 \mathrm{Ca}^{2+}$ troponin-C.troponin-I derived from small angle scattering data: Implications for regulation," Biochemistry 33, 12800-12806 (1994).

[8] Dyson, J. H., Rance, M., Houghten, R. A., Lerner, R. A., Wright, P.E. "Folding of immunogenic peptide fragments of proteins in water solution. II. The Nascent Helix," J. Mol. Biol. 201, 201-217 (1988a).

[9] Campbell, A. P., Sykes, B. D. "Interaction of troponin I and troponin C. Use of the two-dimensional nuclear magnetic transferred nuclear Overhauser effect to determine the structure of the inhibitory troponin I peptide when bound to skeletal troponin C," J. Mol. Biol. 222, 405-421 (1991). 